\title{
MMNET: A Multi-Modal Network Architecture for Underwater Networking
}

\author{
Jun Liu ${ }^{1,2}$, Jun Wang ${ }^{3}$, Shanshan Song ${ }^{1, * \mathbb{C}}$, Junhong Cui ${ }^{1}$, Xiaoyu Wang ${ }^{1}$ and Benyuan Li ${ }^{1}$ \\ 1 College of Computer Science and Technology, Jilin University, Changchun 130012, China; \\ liujun1509@jlu.edu.cn (J.L.); junhong_cui@jlu.edu.cn (J.C.); wangxiaoyu18@mails.jlu.edu.cn (X.W.); \\ liby18@mails.jlu.edu.cn (B.L.) \\ 2 College of Electronic Information Engineering, Beihang University, Beijing 100191, China \\ 3 College of Software, Jilin University, Changchun 130012, China; wjun18@mails.jlu.edu.cn \\ * Correspondence: songss@jlu.edu.cn; Tel.: +86-1594-877-2259
}

Received: 10 November 2020; Accepted: 16 December 2020; Published: 18 December 2020

\begin{abstract}
At present, the key to underwater sensor network (UWSN) research is to provide personalized network support for many underwater applications. In order to achieve this goal, people need a general UWSN. Most of the current UWSN architecture is based on the traditional network, which are limited to a single hardware platform and software platform. Facing the current numerous underwater applications and heterogeneous networks, the UWSN is unable to provide personalized network services according to different application requirements. In this paper, we propose a heterogeneous network framework called MMNET (multimodal network) based on the idea of multimodality, aiming to achieve the compatibility of heterogeneous networks and the scalability of the new architecture. In addition, in the face of the complexity of heterogeneous networks and the personalized needs of network applications, the resource allocation is expressed as a personalized recommendation problem. The distributed personalized recommendation algorithm is used to configure personalized network resources for applications. Each node only needs to solve its own problems, instead of exchanging channel state information by using a distributed algorithm, so the computational complexity can be greatly reduced and signaling is overhead. Finally, we give a special example to prove that our network framework provides a good application.
\end{abstract}

Keywords: multi-modal; heterogeneous network; under-water sensor network; personalized network resource allocation

\section{Introduction}

\subsection{Motivation}

In recent years, with the development of underwater sensor networks (UWSNs), there have been great achievements in the fields of underwater environment monitoring, marine data collection, underwater positioning, offshore exploration, disaster prevention, tactical monitoring and so on. In addition, UWSN is also an important support for autonomous underwater vehicle (AUVs) and unmanned underwater vehicle (UUVs) in underwater collaborative navigation and detection. With the increase of underwater sensor application scenarios, the demand for diversification and personalization of UWSNs is also increasing. However, so far, no good framework can provide complete support for complex underwater applications. In order to provide complete support for underwater applications, various new UWSN architectures emerge endlessly [1-6]. These developments have made UWSN more and more heterogeneous and dynamic. For example. 


\subsubsection{Difficult Communication}

In the past few years, people have focused on acoustic communication for UWSNs. However, although the transmission distance of acoustic communication in underwater communication is longer than that of electromagnetic wave, acoustic communication has the characteristics of long propagation delay, high bit error rate and low transmission bandwidth. These limitations of acoustic communication have seriously affected the performance requirements of UWSNs [6,7]. In recent years, new underwater communication methods such as magnetic induction (MI) and light wave have been used to expand UWSNs to meet the needs of UWSNs. Although MI and optical solve the characteristics of acoustic communication, such as long propagation delay and low transmission bandwidth, MI is seriously attenuated in water, and the communication distance is only tens of meters. Optical transmission distance is larger than MI, which can reach hundreds of meters. However, it is interfered by light scattering and ambient light, and the transmission distance is far less than acoustic communication. The existing underwater communication methods cannot meet the needs of underwater networking (Table 1). However, the combination of different communication modes can better meet the communication requirements of underwater networks. Therefore, an UWSN framework supporting heterogeneous communication of various hardware is needed. Most of the existing underwater multi-mode communication frameworks are based on the adaptation layer, and need a special routing algorithm to support, but cannot achieve the versatility, re-programmability and transparency of multi-mode communication [7-14].

Table 1. Characteristics of underwater MI (magnetic induction), acoustic, and optical communications.

\begin{tabular}{|c|c|c|c|c|}
\hline Media & $\begin{array}{l}\text { Propagation } \\
\text { Speed }(\mathrm{m} / \mathrm{s})\end{array}$ & $\begin{array}{c}\text { Data } \\
\text { Rates/bps }\end{array}$ & Distance/km & Media Dependency \\
\hline MI & $3.33 \times 107$ & $\sim \mathrm{M}$ & $0.01 \sim 0.1$ & Conductivity \\
\hline Acoustic & 1500 & $\sim \mathrm{K}$ & $1 \sim 20$ & $\begin{array}{l}\text { Multi-path, Doppler, temperature, } \\
\text { pressure, salinity, environment } \\
\text { sound noise }\end{array}$ \\
\hline Optical & $3.33 \times 107$ & $\sim \mathrm{M}$ & $0.01 \sim 0.1$ & $\begin{array}{l}\text { Light scattering, line of sight } \\
\text { communication, ambient light noise }\end{array}$ \\
\hline $\begin{array}{l}\mathrm{RF}(\text { Radio } \\
\text { Frequency) }\end{array}$ & $3.33 \times 107$ & $\sim \mathrm{M}$ & $0.01 \sim 0.6$ & Attenuation \\
\hline
\end{tabular}

\subsubsection{Difficult Route Forwarding}

The routing and forwarding of traditional UWSN depends on the node address. Even though the new software-defined network architecture (SDN) is based on the node address in the form of flow table to forward the packets $[7,9,13-16]$. However, in UWSN, there may be such a situation as: Other underwater nodes in the same area may have the latest sensing data of the area, and the data consumers can ignore the information of the nodes themselves. Unlike using the node address to aggregate the sensing data, this scenario can use the data name to request the data, regardless of which node satisfies the data request. The requesting node requests data or content by sending a message containing the desired content name. Using names to represent data helps packets identify and discover different parameters or components of data [17-22]. When any node with the required data receives the message of interest, it only needs to send the data message containing the required data. However, the existing network based on node address cannot support this routing and forwarding method based on name very well. Recently, people have proposed underwater named data networking (NDN) network architecture for this scenario, but these architectures are only designed for content-based routing and cannot support other routing methods, such as geographic-location-based routing. In order to meet the needs of various underwater routing methods, an extensible UWSN framework supporting multiple routing modes is needed. On the Internet, a variety of scalable network architectures are proposed to support different routing and forwarding, and new routing and forwarding protocols can 
be extended smoothly. With the development of underwater applications, UWSNs also need scalability and corresponding capabilities.1.1.3. Applications That Are Difficult to Organize

The current UWSN systems are usually application-oriented, and the system designed for one application cannot be applied to other applications [1-3,5]. With the development of underwater applications, the traditional application-oriented UWSN architecture has been unable to meet the increasingly complex requirements of underwater tasks. The existing network architecture cannot meet the needs of all tasks in a unified way. Under the support of the same network architecture, multiple applications interfere with each other, and cannot provide personalized network resources for each application, resulting in the application of each other to seize network resources, resulting in the waste of network resources, and may affect the normal operation of the entire network architecture function. Therefore, we need a network framework that can allow multiple underwater applications to run simultaneously on the same physical UWSN system without interfering with each other.

\subsubsection{Network Management Difficult for Application Requirements}

The current UWSNs are application-oriented. Even the emerging UWSN architecture based on software-defined radio (SDR) and network function virtualization (NFV) only allocates the corresponding network resources to the application, and cannot realize the personalized network resource allocation based on the application requirements. Due to the characteristics of the multi-mode network, the traditional resource allocation model cannot make full use of the advantages of multimodal network, but also cannot meet the personalized needs of network resources [10,11]. Therefore, a network management program that can adapt to multimodal and heterogeneous networks and provide differentiated network resource services is needed.

\subsection{Contribution}

MMNET, with the idea of multimodality, uses NFV and SDR functions to support heterogeneous communication, multi-mode routing and forwarding and multi-application support within a unified architecture, while meeting the networking requirements of diversified and personalized underwater applications [7,9].

Heterogeneous communication. On the basis of general hardware, MMNET connects communication equipment with standard interface, describes different communication modes in the form of software, and provides physical and medium access control (MAC) layer functions for upper layer network protocol in the form of a unified interface, so as to reduce the difficulty of heterogeneous communication caused by different communication modes due to hardware differences, and provide better heterogeneous communication support for UWSN [9-11,14,15,23].

Multi-mode routing and forwarding. MMNET realizes the network routing and forwarding function in the form of software. It virtualizes different network foundations in the network layer and runs different routing and forwarding modes at the same time, which can meet the personalized requirements of different routing methods for UWSN at this stage and provide more networking options for UWSNs [24-27].

Multi-application organization. MMNET uses virtualization technology to create multiple virtual networks. Each virtual network hosts different applications and runs under its own application. It does not conflict with other applications to meet the personalized needs of different applications.

Intelligent network management is backed on application requirements. In the face of the complexity of a multi-mode heterogeneous network and the personalized demand of network resources for network applications, resource allocation is expressed as a personalized recommendation problem. The distributed personalized recommendation algorithm is used to configure personalized network resources for applications. The advantages of a multimodal heterogeneous network are fully utilized to provide personalized network support for underwater applications [27].

The rest of this paper is arranged as follows. In the Section 2, a new network architecture MMNET is proposed, and the basic principles and key technologies of the network architecture are introduced in 
detail. The Section 3 will introduce the intelligent network management algorithm based on application requirements. In the Section 4, a specific case is studied to further illustrate the working process of network architecture. Finally, the Section 5 summarizes the thesis.

\section{Network Architecture}

In this section, we will introduce the architecture of MMNET (Figure 1), a network architecture based on multi-modal concept, and describe the relevant technical advantages. According to the characteristics and shortcomings of the existing UWSN framework, combined with the existing network technology, the overall design idea of MMNET is as follows.

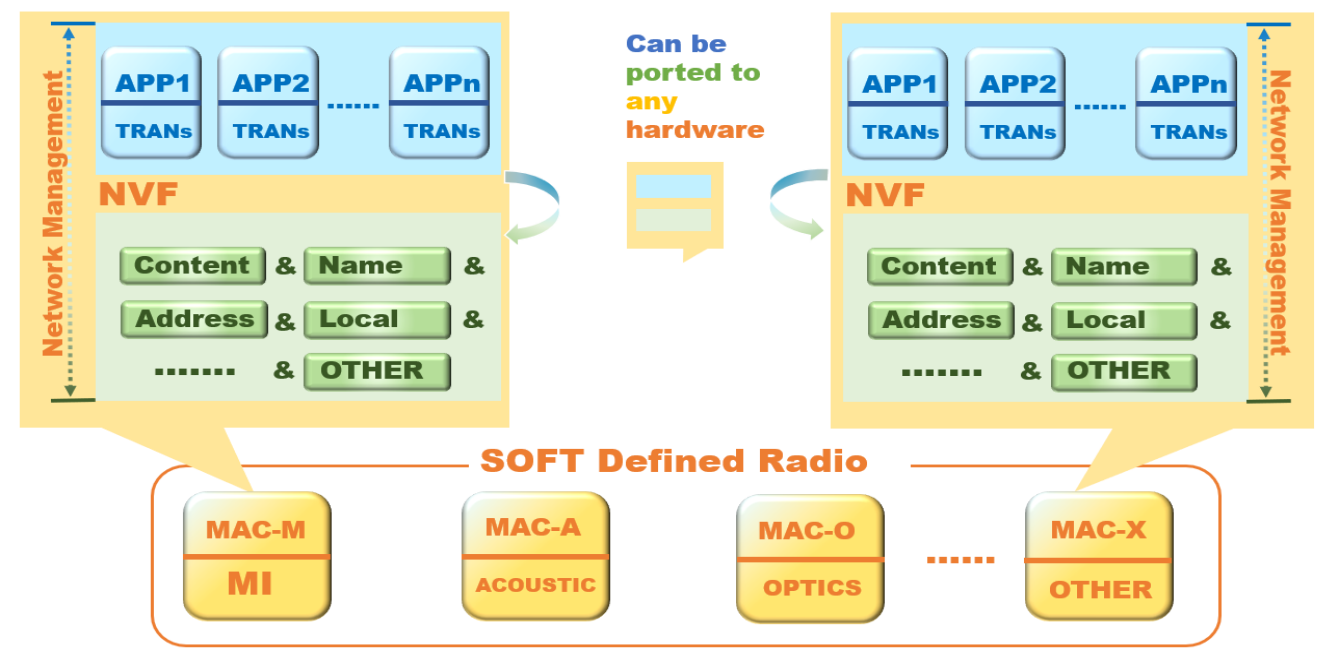

Figure 1. The architecture of MMNET (multimodal network). MMNET uses software defined radio (SDR) technology and standard hardware interface to realize multi-mode communication, including multimedia access (MAC) layer and physical layer, and uses network function virtualization (NFV) technology to organize application (APPs) layer, transmission layer (Trans) and routing layer.

\subsection{Multimodal Access Interconnection in MMNET}

In order to better support multi-mode heterogeneous communication and use multi-mode communication technology to support applications, MMNET adopts a standard hardware interface and SDR to realize physical layer and MAC layer; the standardized hardware interface is to better support different hardware front ends. SDR aims to support differentiated communication technology, on the basis of the same hardware architecture, and provide a transparent communication interface for the whole network.

In MMNET, different hardware front ends are connected to general hardware through a standard hardware interface (such as serial port, bus interface, etc.); the standard hardware interface is conducive to supporting more hardware devices. New communication hardware can be supported on the whole network as long as it meets the requirements of the hardware interface. When the communication hardware is used, SDR technology can be used to realize the software implementation of the new communication mode. On the premise of not replacing the hardware front end, it can provide updated and more communication technology support, reduce the cost of network use, improve the performance of the whole network and provide more communication support for applications.

Using SDR technology, MMNET encapsulates different communication technologies into communication services by software and provides a unified communication interface for the network. When new communication technology is needed, new communication equipment is connected on the basis of general hardware, and the communication method, MAC protocol and service interface are realized by software. After the transparent network communication service interface is implemented, the network communication service interface will be registered in the network 
management program and the new communication technology will be supported in the current network. This function of MMNET, as shown in the Figure 2, can enhance the communication ability of the underwater network and improve the performance of the whole network by using different underwater communication equipment, such as MI, optical harmony and Orthogonal Frequency Division Multiplexing (OFDM) technology.

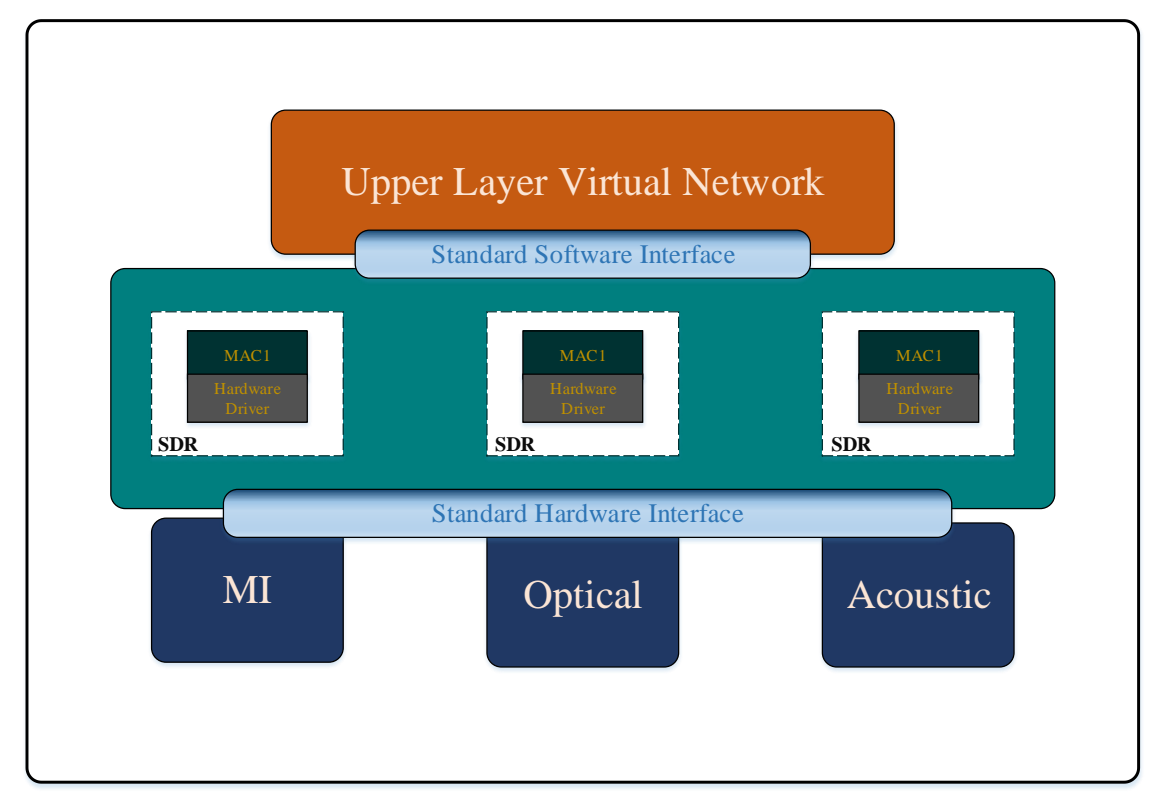

Figure 2. Implement of multi-modal access interconnection in MMNET.

\subsection{MMNET Multimodal Routing and Forwarding}

In order to provide personalized underwater network services, MMNET will provide multimodal addressing and routing methods to meet the personalized addressing requirements of applications, provide various addressable and routing capabilities that can be defined, and support multiple converged network addressing and routing considering the complex application scenarios and requirements of underwater applications.

As shown in the Figure 3, in MMNET, different routing protocols are run on a common hardware platform by using network function virtualization. Different routing protocols run on the same hardware platform in the form of virtual machine (vnf), ignoring the hardware requirements of traditional routing protocols, so that MMNET can better support different routing protocols. With the support of NFV, different routing protocols can run in the same network architecture in parallel. Different routing protocols will not affect each other because of hardware resource occupation. They can provide different routing and forwarding capabilities for different applications in parallel [7,27]. In MMNET, the virtualization routing protocol communicates with the lower virtualization communication equipment and the upper virtualization application through a fixed interface. At the same time, because MMNET ignores the hardware requirements and connects with the upper and lower layers through fixed interfaces, it can well extend the support of the new architecture. 


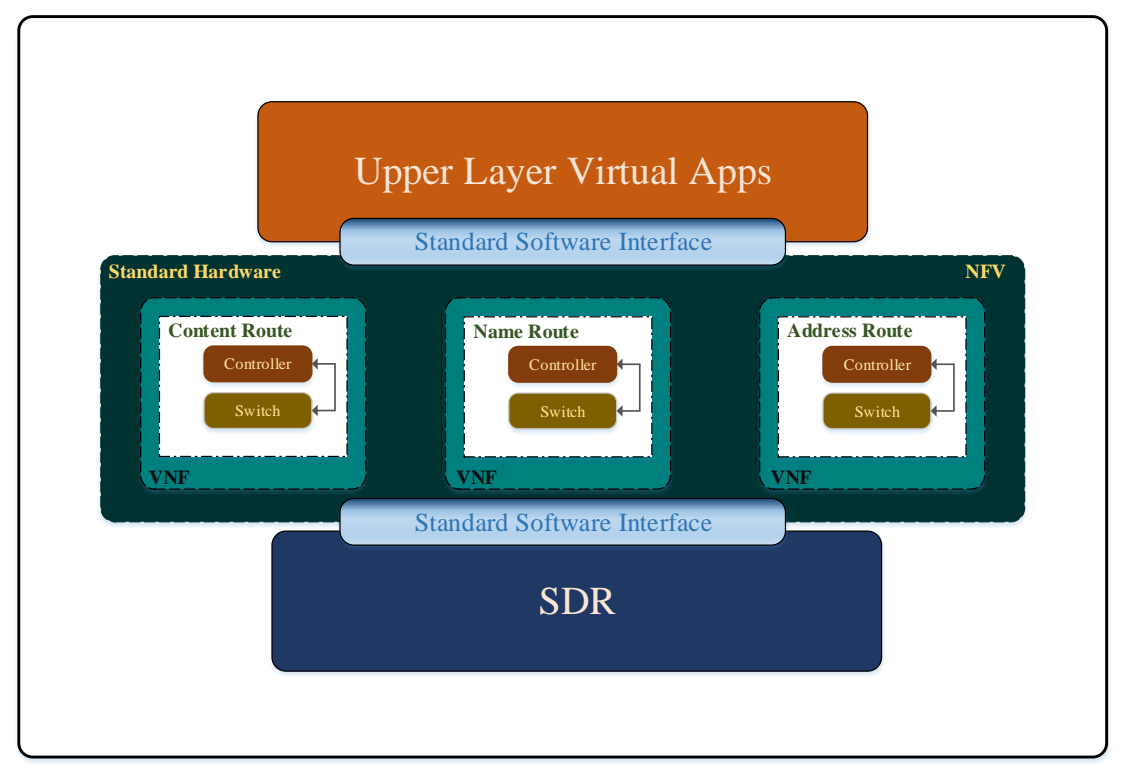

Figure 3. Implement of MMNET multi-modal routing and forwarding. Each route is organized in the form of virtual machine (vnf), which does not affect each other and runs independently.

In MMNET, considering the harsh underwater communication environment and tight communication resources, as well as better scheduling routing protocol, the routing control function and forwarding function are concentrated in the same VNF, as shown in Figure 4. As shown in Figure 5, the separation of network control and forwarding can make better use of global information for better routing scheduling in terrestrial networks. However, in UWSNs, the limitations of existing communication modes, such as long propagation delay and valuable communication bandwidth of acoustic communication, and short communication distance between optical and magnetic communication will seriously affect the sending and receiving of control information, so as to affect the actual performance of the whole network. At the same time, compared with the traditional network architecture, the simultaneous operation of multiple routing protocols further increases the exchange of routing control information, which seriously affects the performance of the whole network. If the control function and forwarding function are integrated into VNF, it will lose the guidance of global information on forwarding strategy, but it can effectively save bandwidth resources and improve the response speed of the control algorithm. At the same time, a variety of routing algorithms complement each other, which will effectively reduce the loss of a single routing algorithm due to the lack of global information. 


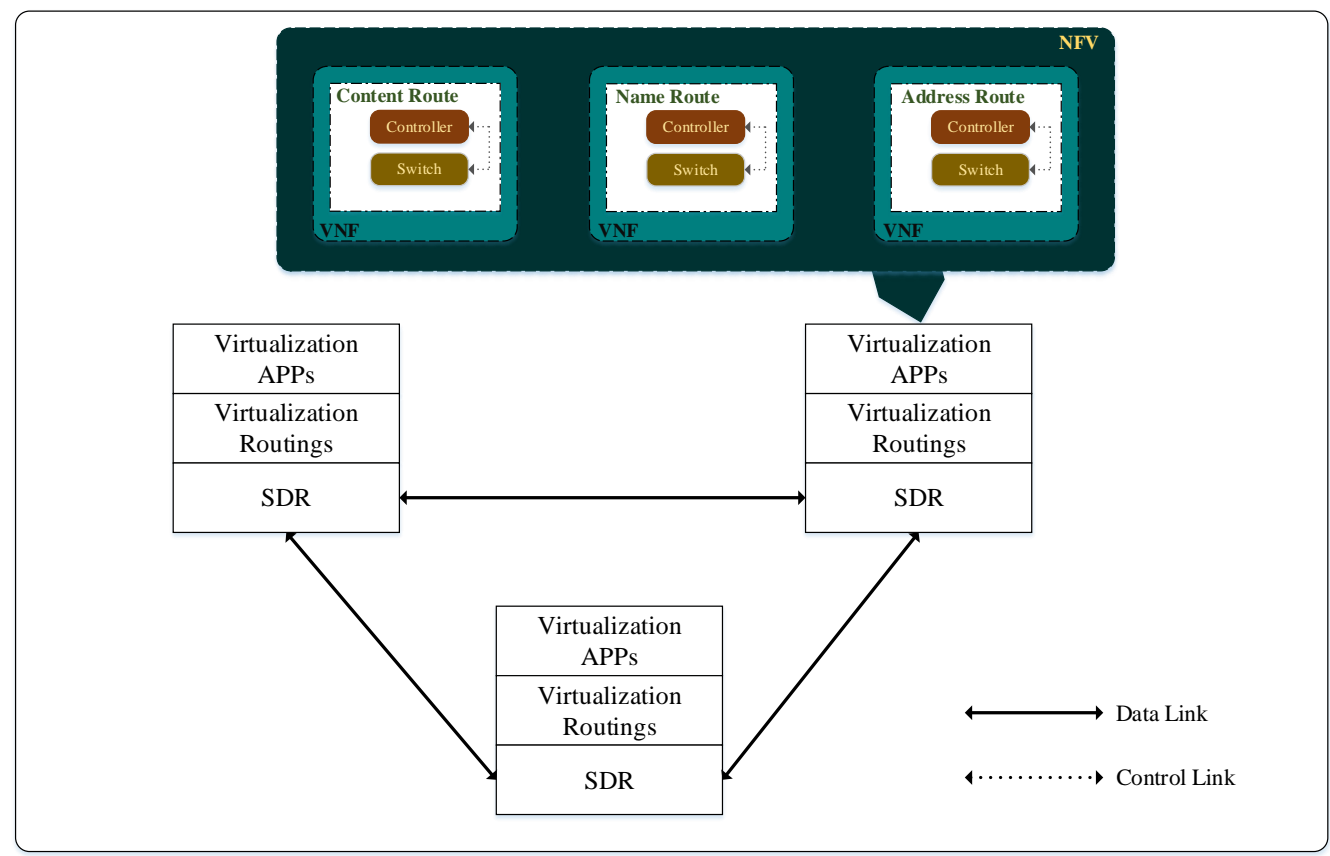

Figure 4. Implement of control forwarding of MMNET routing.

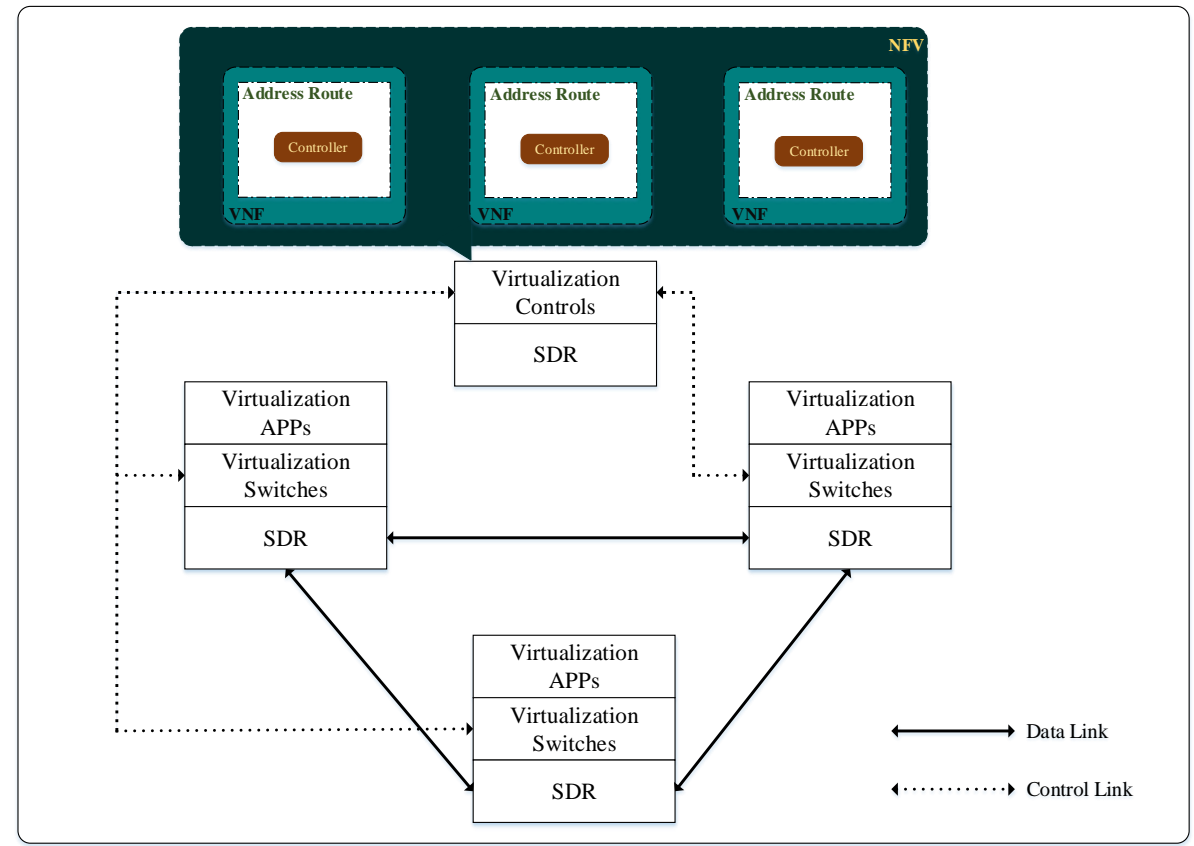

Figure 5. Routing implementation of control forwarding separation.

\subsection{MMNET Multi-Application Organization}

With the increasing demand for underwater applications, the number and types of applications that need to be carried by UWSNs is increasing, and it is more difficult for different underwater applications to propose UWSN architecture for application organization. Researchers have carried out a variety of explorations based on traditional UWSN architecture, which cannot solve the organizational problems between different applications $[1,6,7,27]$. As shown in Figure 6, NFV virtualizes the traditional hardware-based functions, integrates the physical resources and provides them to multiple applications, so that each application can share the physical resources while isolating from other applications, and each application does not interfere with each other in space and time. Through NFV, MMNET can make many different applications share network resources. 


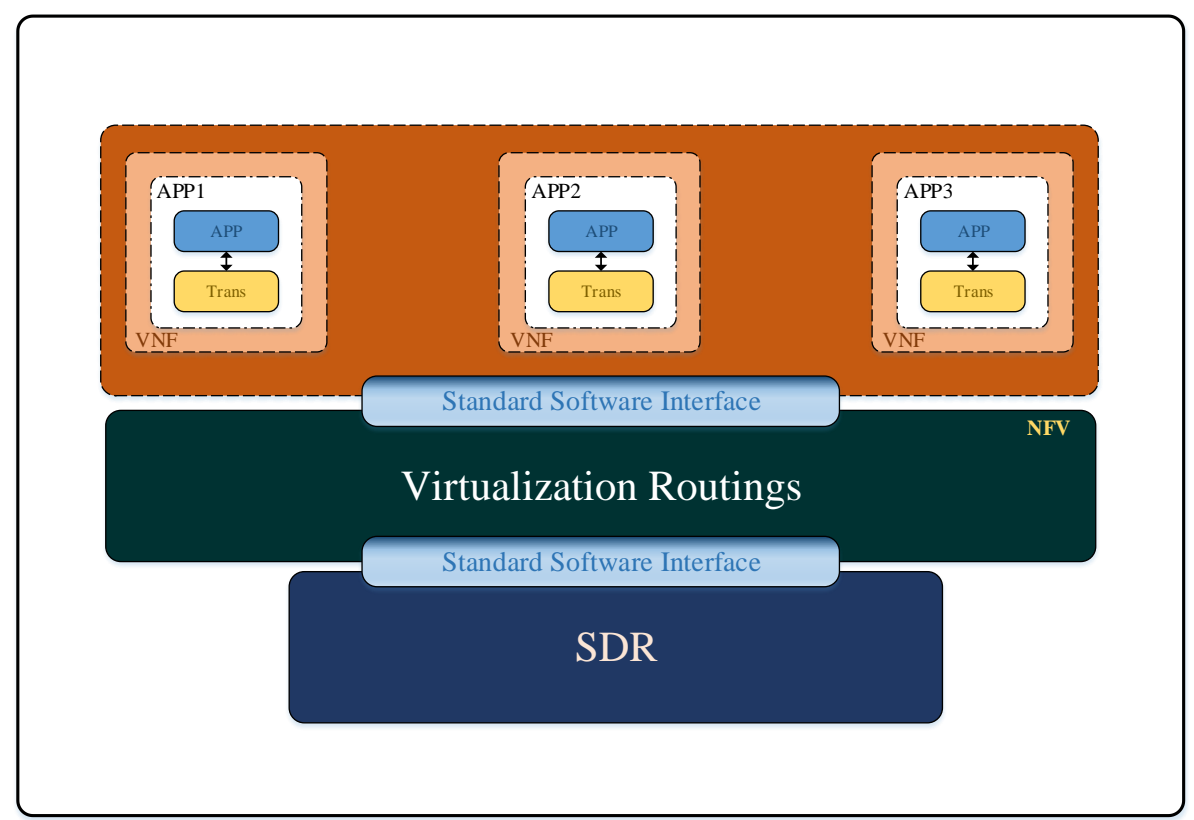

Figure 6. Implement of MMNET multi-application organization.

Each application can also choose its own physical layer, MAC layer and net layer according to their own needs, which can not only meet the diversified personalized needs of each application for the network, but also achieve the mutual non-interference among multiple networks, thus solving the organization problem of network applications.

\section{Intelligent Network Management Based on Application Requirements}

\subsection{MMNET Personalized Network Resource Allocation}

In UWSNs, the application scenarios and types are complex, and each application needs different network resources and functions, so it is necessary to provide personalized network support for applications [22,28-30].

In order to provide personalized networks for different applications, considering the specific situation of MMNET, we propose a personalized network support scheme based on K-nearest neighbor $(\mathrm{KNN})$. The main idea of this method are as follows: Firstly, according to the type of task, the network requirements are extracted. Secondly, according to the extracted requirements, KNN is used to recommend the application scheme of network resources based on historical data.

\subsubsection{Network Demand Extraction}

As shown in Figure 7, according to the characteristics of the current task, the application sends its task feature matrix to the requirement extraction algorithm, which will calculate the demand matrix of the current task according to the historical data.

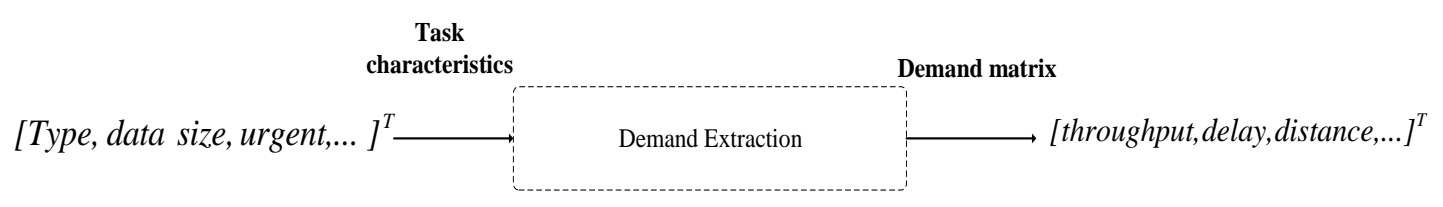

Figure 7. Network requirements extraction process.

\subsubsection{Personalized Network Resource Application Based on KNN}

As shown in the Figure 8, the task requirement matrix extracted according to the task is sent to the $\mathrm{KNN}$ recommendation algorithm, and the KNN algorithm will recommend the most qualified network 
resource application scheme in the top $\mathrm{n}$ according to the historical data. As shown in Figure 9, after obtaining the recommendation matrix of network resources, the transport layer of the application will apply to the network management program for resources from the first type according to the recommendation matrix. When the first application fails, it will apply for the second scheme, and after the second fails, the third scheme will be tried until all the recommended schemes are tried. When the scheme application is successful, the characteristic matrix of the current scheme and the successful scheme matrix will be added to enrich the historical data.

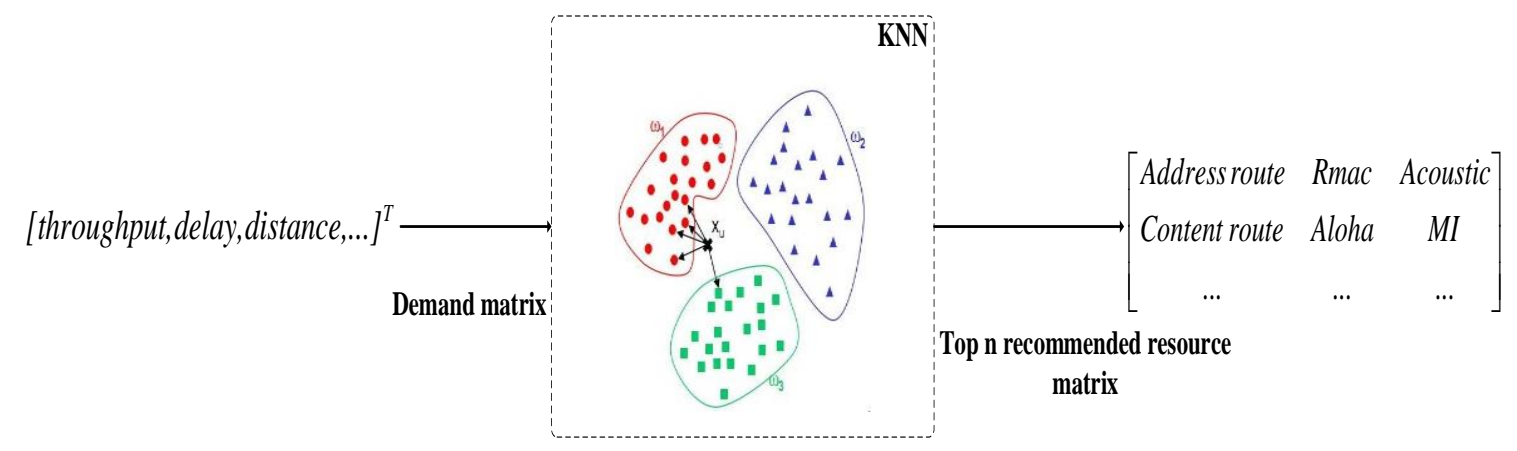

Figure 8. Recommend network configuration based on requirements.

0.Select requirements from the requirements matrix

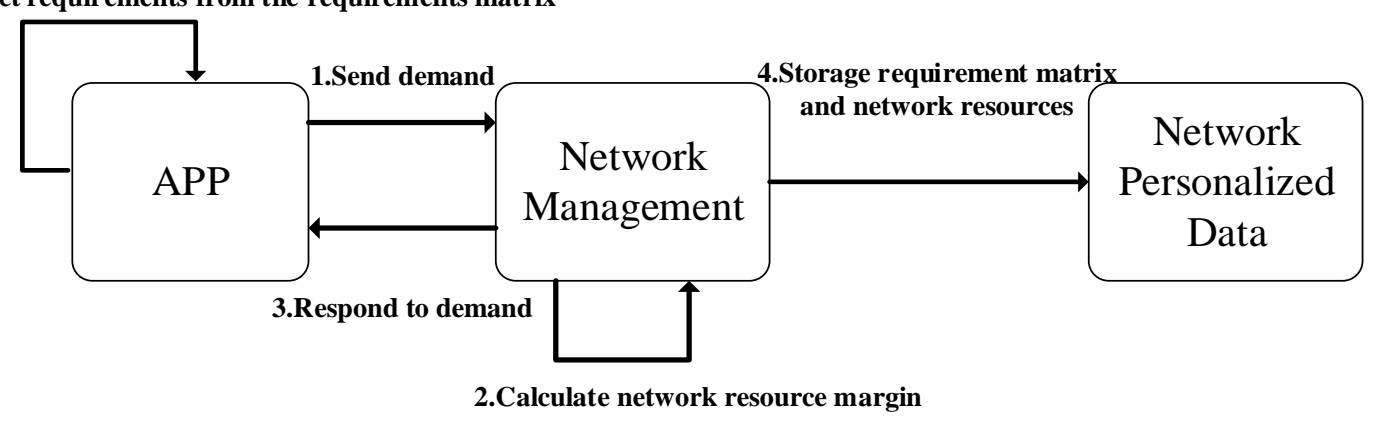

Figure 9. Apply for resources according to the recommended network configuration.

\subsection{MMNET Network Management}

At present, although there are many kinds of underwater communication technologies, there are still some limitations, which cannot be used as the information forwarding control of network management. First of all, acoustic communication has the characteristics of long propagation delay, high bit error rate and low transmission bandwidth. If it is used to manage the network, it will not be able to timely transfer the status of the network and occupy valuable communication bandwidth. Although underwater optical communication and underwater magnetic communication overcome the characteristics of high delay and small bandwidth of acoustic communication, their short communication distance limits the coverage of the network. The limitations of various underwater communication technologies affect the centralized network management mode in an underwater network. In view of this feature, MMNET chooses the distributed network management mode. Each node manages its own network and reduces the transmission of control information. It can not only enhance the sensitivity of network control, but also reduce the occupation of communication bandwidth and expand the coverage of the network. Two major functions of MMNET network management are as follows.

\subsubsection{The Addition of Virtualization Network Function}

As shown in Figure 10, when a new virtualization device or function wants to join MMNET, the new device first sends the join request information to MMNET. After receiving the join request, the network management program allocates a unique ID for a period and sends its unique ID to the 
requesting device or function. At the same time, the network manager adds its description array to the personalized network recommendation matrix and allocates its resources when new functions are applied.

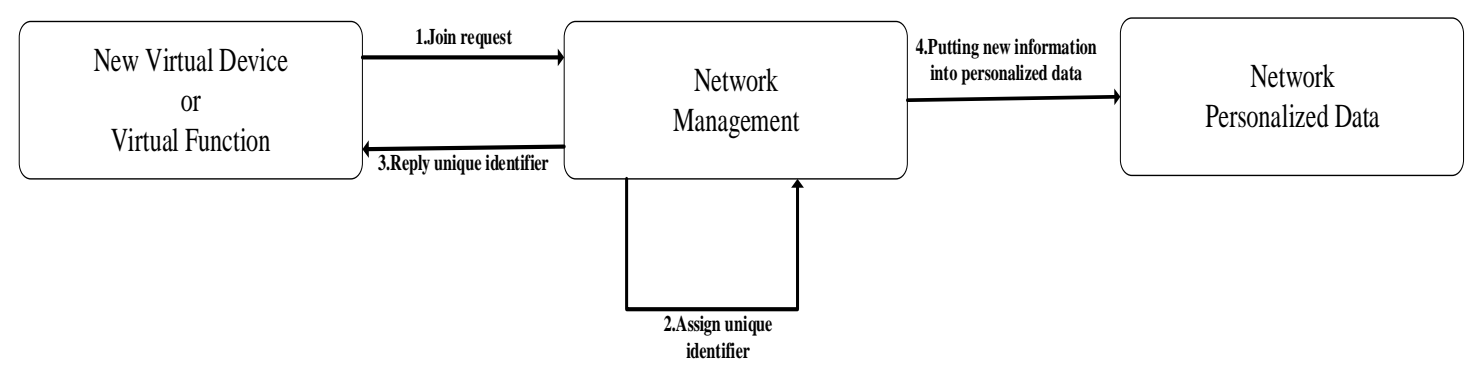

Figure 10. New virtual devices or functions join the network.

\subsubsection{The Allocation of Network Resources}

As shown in Figure 11, when the application applies for resources according to the recommendation information, the network management program will allocate network resources to the application according to the existing network resources, the network management program will reply with the ID of relevant resources to the application, and the application will use the applied resources according to the obtained ID. When the current resources cannot meet the requirements of the application, the network management program will return the application failure message to the application.

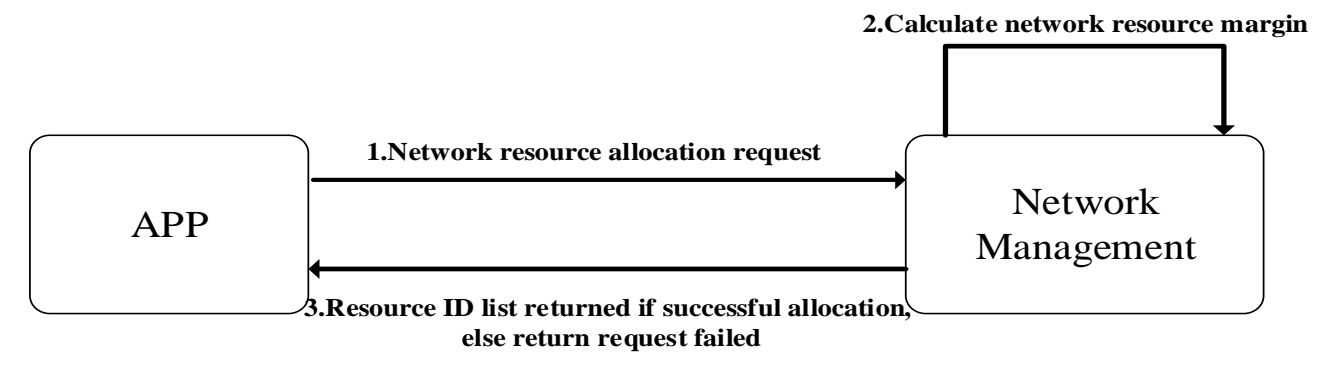

Figure 11. The network manager allocates network resources to applications.

\section{Study Case}

Through the introduction of MMNET, we have a certain degree of understanding of MMNET recommendations for UWSNs. Here is a study case to deepen our understanding of this.

As shown in Figure 12, there are two types of underwater scenes. One is the underwater environment monitoring (completed by fixed sensor nodes) and the other is underwater data collection. AUV is mainly responsible for underwater data collection.

Underwater environmental monitoring includes two types of forwarding networks and two types of heterogeneous networks. The Figure 13 shows the network equipment and network protocol carried by each network node, and its communication process. 


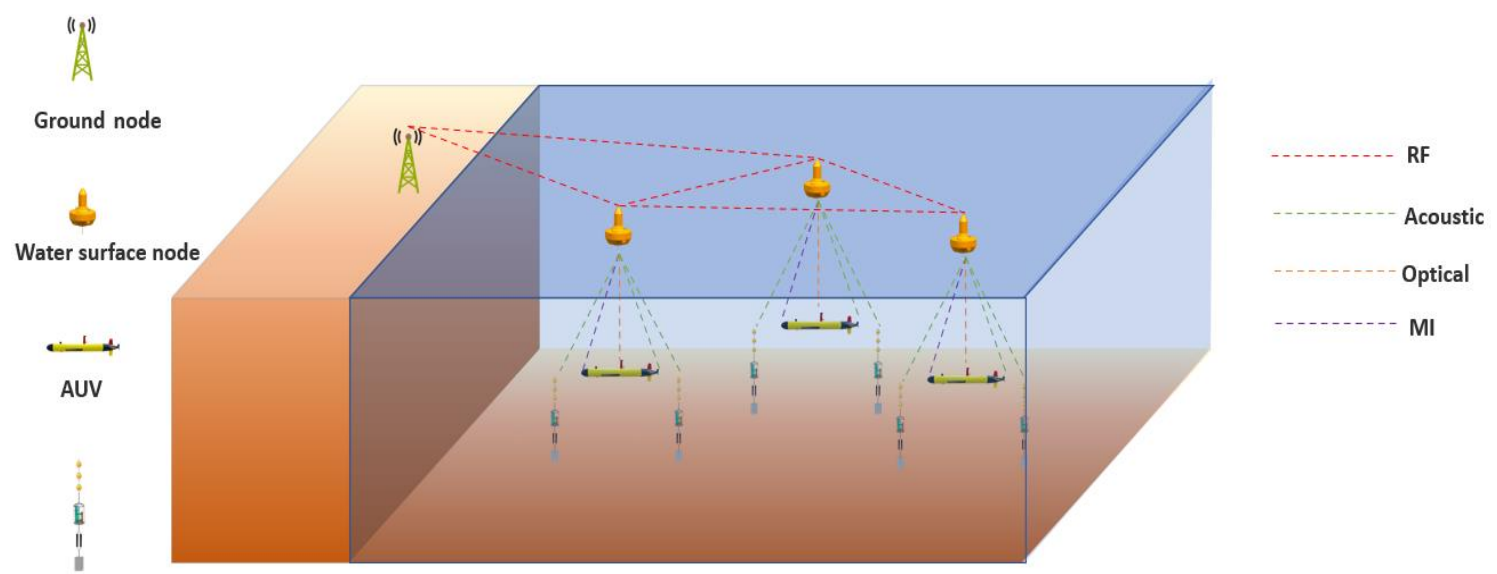

Underwater node

Figure 12. Case study for the MMNET. This study case includes four kinds of nodes: Ground node, water surface node, autonomous underwater vehicle (AUV) and underwater node. Each node runs one or two applications (APPs). Each node may carry radio frequency, acoustic, optical and magnetic induction (MI). At the same time, each node carries address-based routing and name-based routing.

Underwater environmental monitoring includes two types of forwarding networks and two types of heterogeneous networks.
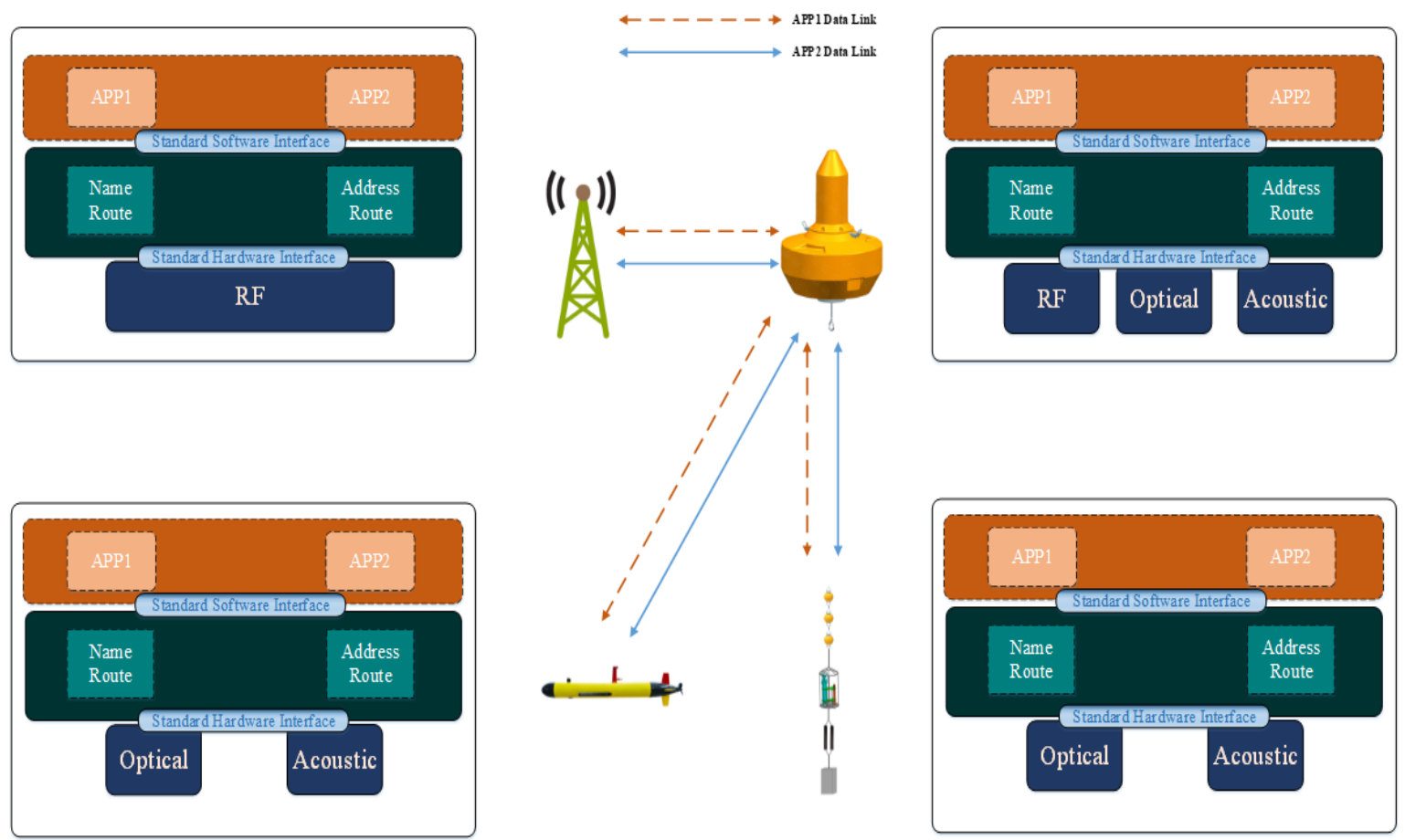

Figure 13. Status and information of each network node.

As shown in Figure 14b, in order to monitor the underwater environment information, fixed nodes will be equipped with CTD, temperature and salinity depth, dissolved oxygen and other sensors. These sensor data are triggered by timing tasks. After the timing tasks are triggered, as shown in Figure 14a, the network management program will extract the characteristics of the tasks, recommend the network resources according to the characteristics of the tasks, and then apply for network resources according to the recommended network configuration and configure them. After the network configuration is completed, the underwater environment information will be uploaded 
according to the time. After receiving the data from the underwater, the water node will send the wireless telex to the surface application.

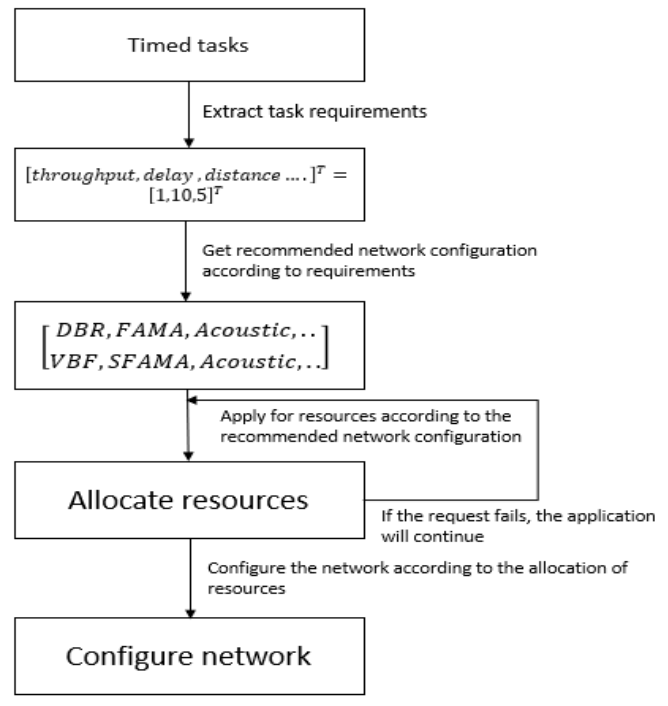

(a)

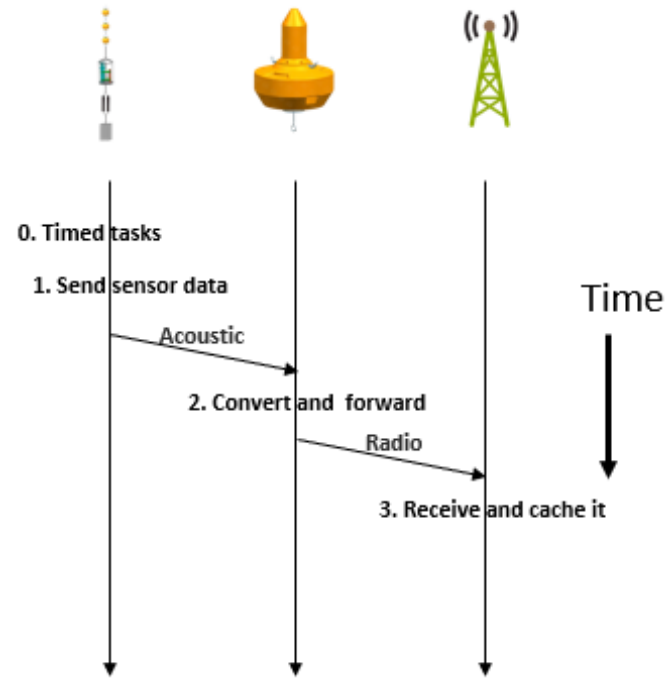

(b)

Figure 14. (a) Application process of network resources for timed tasks; (b) detailed information of interaction between timed task terminals in underwater environment monitoring network.

As shown in Figure 15b, when the underwater environment information changes, in order to confirm the specific changes of the underwater environment, as shown in the Figure 15a, the network management program will extract the characteristics of the task, recommend the network resources according to the characteristics of the task, then apply for the network resources according to the recommended network configuration, configure the network to be named based on the routing and forwarding strategy, and send interest After receiving the interest packet, the underwater node will upload the data package that meets the requirements to help the surface application to study and judge the environmental conditions.

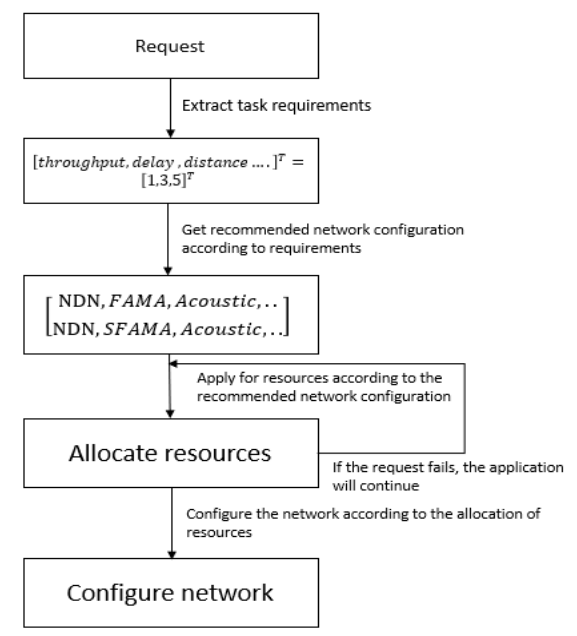

(a)

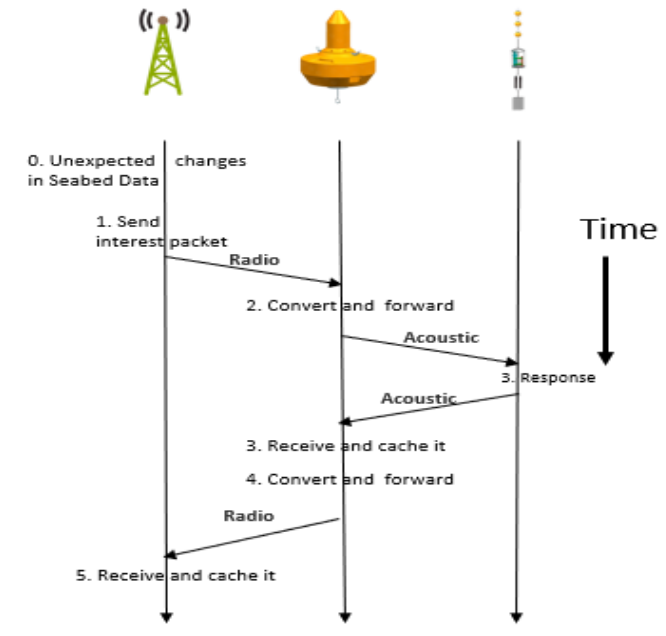

(b)

Figure 15. (a) Emergency data request for underwater detection application; (b) detailed information of interaction between emergency task terminals of underwater environmental monitoring network.

Underwater data collection network includes two kinds of forwarding networks and three kinds of heterogeneous networks. 
As shown in Figure 16b, when the underwater data collection is not urgent, the AUV will cruise underwater to collect data, and the AUV node will locate with the surface node through the acoustic network. After the AUV data collection is completed, as shown in Figure 16a, the network management program will extract the characteristics of the task, recommend the network resources according to the characteristics of the task, and then apply for the network resources according to the recommended network configuration. The data exchange between AUV and the surface node is carried out through optical or magnetic communication. After the information exchange is completed, AUV will continue to collect the underwater data and the surface node will transmit the data to the surface application program through radio.

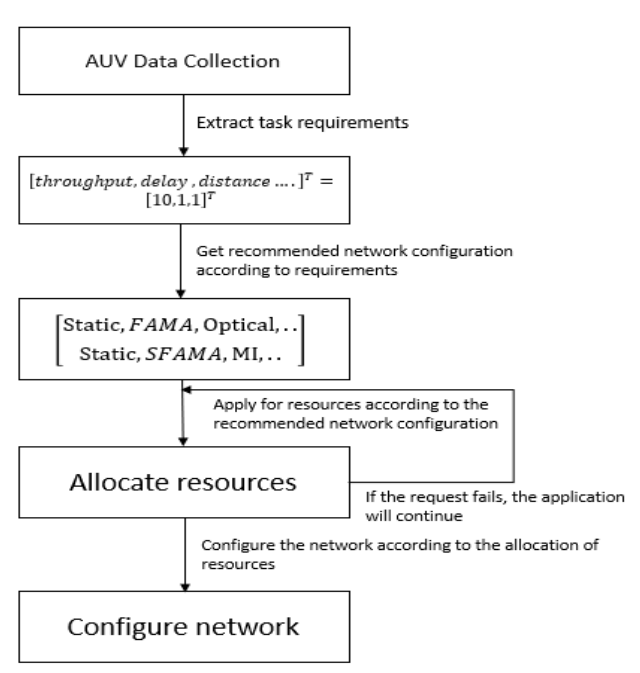

(a)

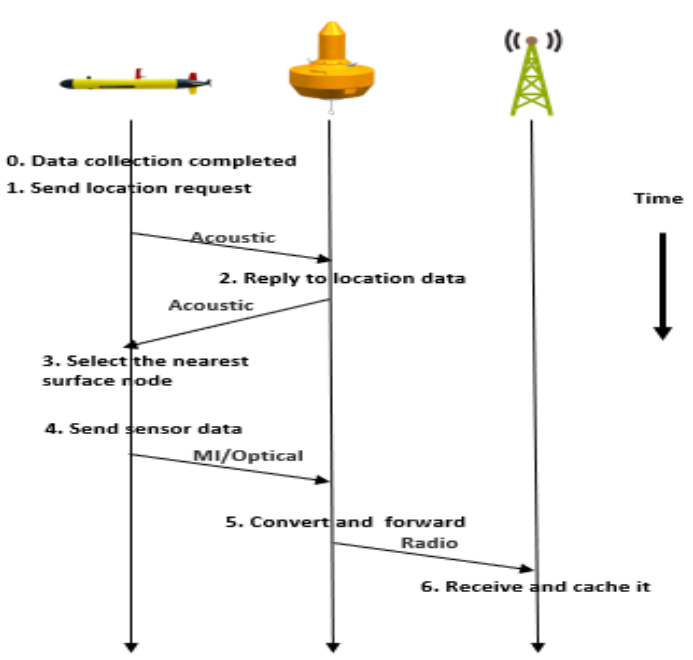

(b)

Figure 16. (a) Data collection application network resource request; (b) detailed information on the interaction between terminals of underwater data collection network.

As shown in Figure 17b, when the surface application is in urgent need of data in a certain area of the seabed, as shown in Figure 17a, the network management program will extract the characteristics of the task, recommend the network resources according to the characteristics of the task, then apply for the network resources according to the recommended network configuration, use the named routing forwarding strategy network to send the interest data packet, and the surface node receives the interest data packet and uses the acoustics. The AUV that has collected the relevant data will respond to the interest data packet. The data packet is sent to the water surface node through the acoustic network, and the water surface node will send the data packet to the surface application program by radio. 


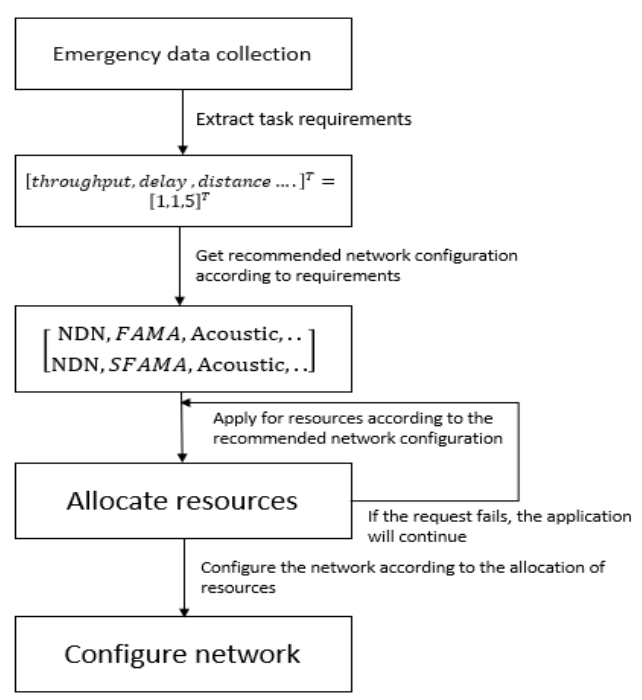

(a)

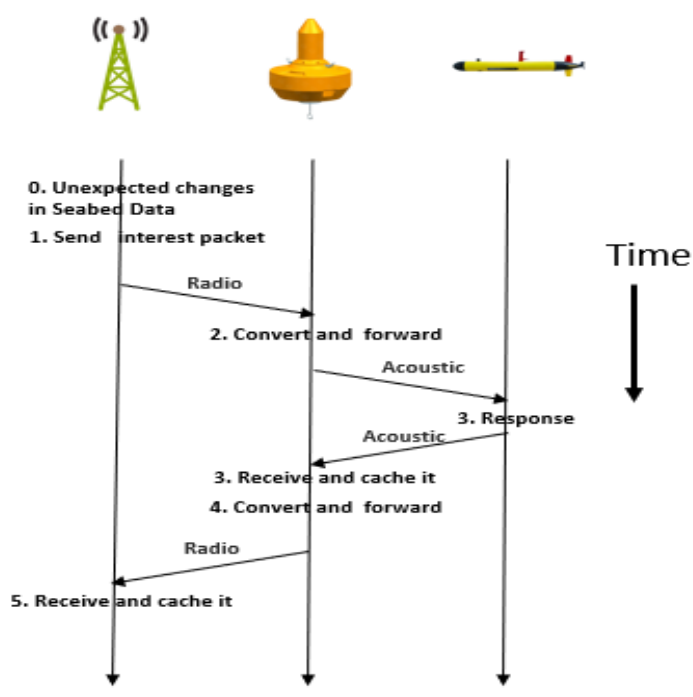

(b)

Figure 17. (a) Data collection application emergency network resource request; (b) detailed information of interaction between terminals of the underwater data collection network emergency task.

\section{Simulation}

In order to evaluate the performance of our proposed MMNET, we simulated the multi-mode routing and multi-mode communication of MMNET respectively. In order to evaluate MMNET's multi-mode communication capability, we considered a classic AUV-assisted underwater data acquisition scenario. Both AUV node and collection node were equipped with acoustic and optical communication equipment. The effective distance of optical communication equipment was $100 \mathrm{~m}$, the packet rate conformed to the Poisson distribution of $\lambda=40$ packets per second, and the effective communication distance of acoustic communication equipment was $1000 \mathrm{~m}$. According to the Poisson distribution of $\lambda=0.4$ packets per second, the AUV collected the underwater environment information from the origin with the graph path, as shown in Figure 18. The yellow path represented the optical communication range, and the blue path represented the acoustic communication range. The simulation was carried out for $310 \mathrm{~s}$.

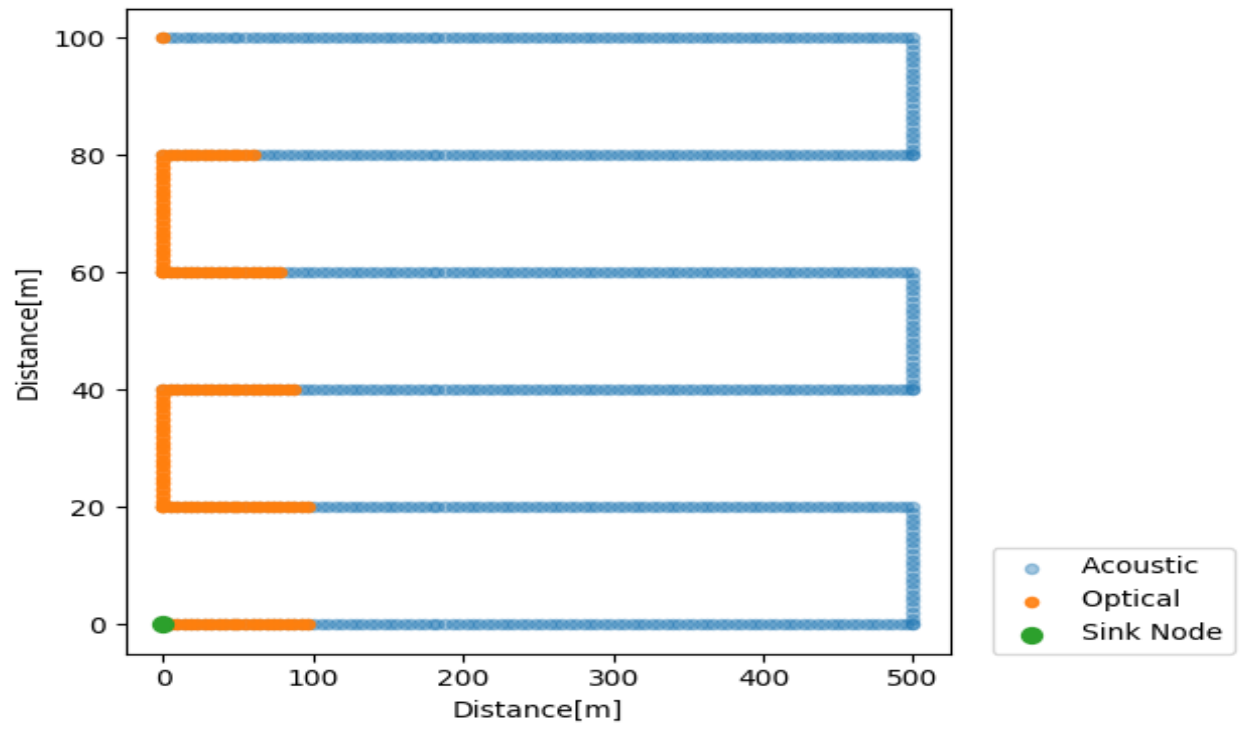

Figure 18. Available bandwidth of AUV trajectory and corresponding position. 
Figure 19a showed the average number of packets per $10 \mathrm{~s}$ of acoustic and MMNET multi-mode communication systems. Since acoustic communication cannot communicate within $100 \mathrm{~m}$, it was not compared with MMNET. It could be seen from Figure 19a that, in the optical communication range, MMNET had a transmission rate much higher than that of acoustic communication. Even if it exceeded the optical communication range, MMNET could still communicate at the same rate as acoustic communication. It could be seen that the performance of MMNET communication was better than that of traditional single mode communication.

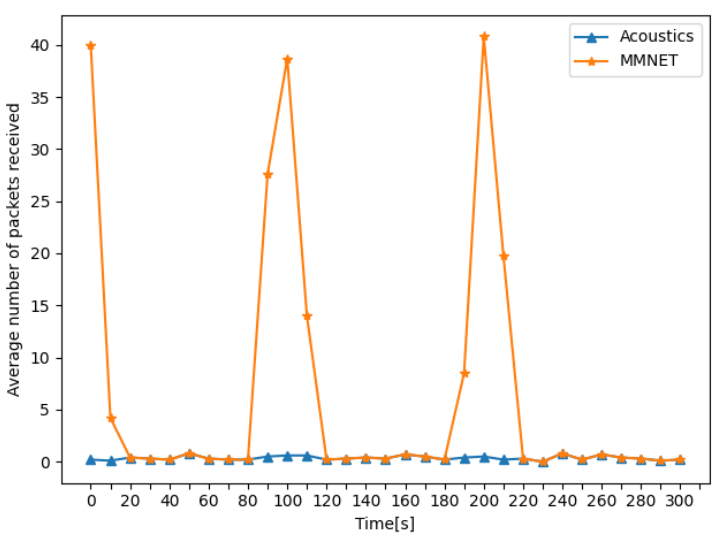

(a)

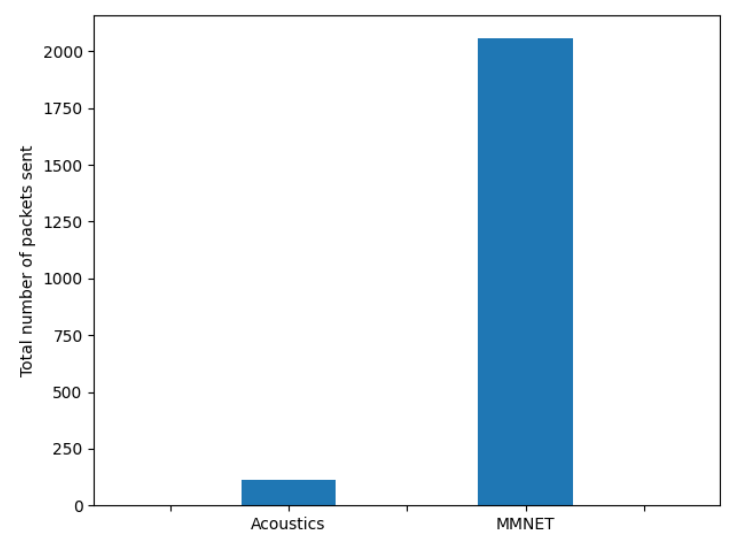

(b)

Figure 19. (a) Showing $10 \mathrm{~s}$ average packet transmission speed of MMNET and acoustic communication; (b) the total number of contracts in the whole simulation process.

In order to evaluate the multi-mode routing capability of MMNET, we considered a classic underwater data acquisition scenario. Multiple acquisition nodes were deployed around a sink node and acoustic communication was used between nodes. The transmission rate of network packets conformed to the Poisson distribution of $\lambda=0.4$ packets per second. Each node used MMNET to deploy address-based routing and NDN-based routing. The simulation time was $600 \mathrm{~s}$. Assuming that the application had special interest in $5 \%$ of the data packets, it needed to be recollected.

Figure 20a-c showed the ratio of the actual received packets to the expected packets when there were three collection nodes, six collection nodes and nine collection nodes, respectively. Because NDN-based packets needed to send interest packets first, they occupy more network resources and were less efficient than MMNET and address-based routing. However, when the application was interested in some packets, the method based on NDN would reduce the sending of interest packets due to its content aggregation function, while the address-based method needed to send interest packets many times. MMNET used two methods at the same time, which was more efficient. As could be seen from the Figure 20a-c, with the increase of nodes, the performance of address-based routing was gradually declining because it did not have aggregation function. The performance of the NDN-based routing method was low because it needed to send interest packets when collecting data. MMNET provided services for applications in various ways, which cpuld meet the needs of applications to a large extent.

Figure 21a-c showed the additional delay of the packets received by the application and expected to be received by the application, in addition to the delay of the network protocol when there were three collection nodes, six collection nodes and nine collection nodes, respectively. In the normal data acquisition scenario, due to the need to send the interest packet first, the NDN-based data packet increased the delay of receiving the expected packet. The address-based method could upload data directly, and the delay was small. However, when the application was interested in some packets, the NDN-based method would reduce the sending of interest packets due to its content aggregation function, while the address-based method needed to send interest packets multiple times. The application using MMNET could use two routing methods at the same time to select the best 
routing method. It could be seen from the figure that as the number of nodes increases, the delay of address-based routing increased gradually when repeated collection was needed due to the lack of aggregation function. MMNET could be used to provide a variety of application services.

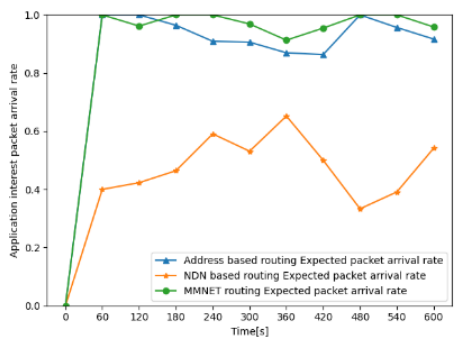

(a)

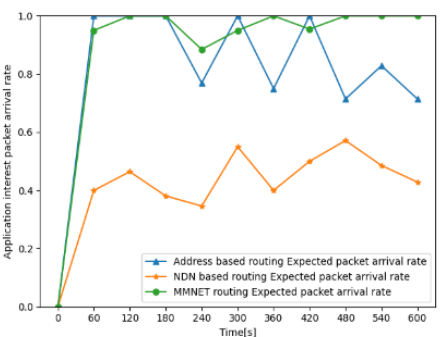

(b)

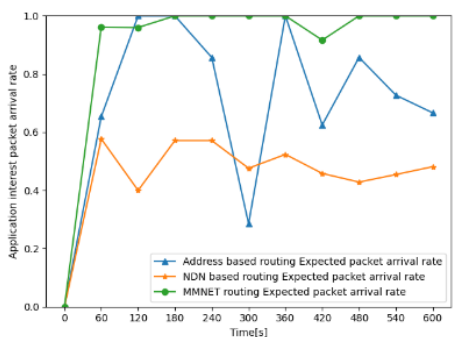

(c)

Figure 20. (a) Application expected packet arrival rate of three collection nodes; (b) application expected packet arrival rate of six collection nodes; (c) application expected packet arrival rate of nine collection nodes.

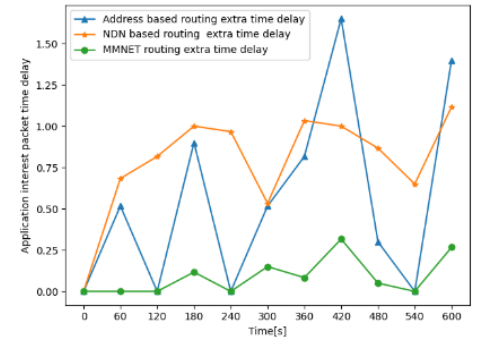

(a)

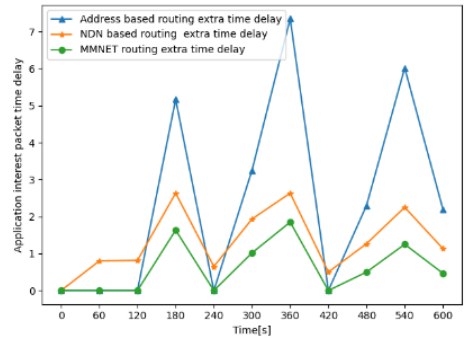

(b)

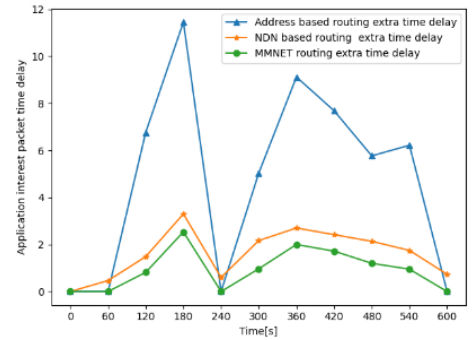

(c)

Figure 21. (a) Application interest packet extra time delay of three collection nodes; (b) application interest packet extra time delay of six collection nodes; (c) application interest packet extra time delay of nine collection nodes.

\section{Conclusions}

As a supplement to the Internet of Things, the underwater sensor network has a broad development prospect. It plays an important role in military communication, environmental monitoring, marine data acquisition and underwater positioning. In order to make better use of the network and further expand the coverage of the Internet of Things, this paper analyzes the characteristics of underwater sensor networks and the challenges of communication, routing and forwarding selection, as well as the application that is difficult to organize. Combined with the characteristics of the existing network architecture, a network architecture MMNET based on multimodality is proposed. MMNET mainly solves the diversified and personalized underwater networking requirements of complex underwater applications at this stage, including the support of heterogeneous multi-mode communication equipment, the support of multi-mode routing and forwarding ability, the organization ability of multiple applications, and the network personalization based on KNN. Through our examples, MMNET will be able to solve some problems of underwater sensor networks at this stage. However, KNN-based personalized network resource allocation can only recommend the existing similar configuration, and cannot provide more personalized configuration for the current configuration. In the future, we will consider strengthening learning and deep learning to optimize the allocation of network resources [27,31].

Author Contributions: Conceptualization, J.L., J.W. and S.S.; methodology, J.L., J.W.; writing, S.S., X.W. and J.W.; writing-review and editing, S.S., J.L., J.W., X.W., J.C. and B.L.; supervision, J.L., S.S. and J.W. All authors have read and agreed to the published version of the manuscript. 
Funding: This work was supported by the National Natural Science Foundation of China (Grants No. 61971206, No.61631008, and No. U1813217), the Fundamental Research Funds for the Central Universities 2017TD-18, the National Key Basic Research Program (2018YFC1405800).

Conflicts of Interest: The authors declare no conflict of interest.

\section{References}

1. Peng, Z.; Zhou, Z.; Cui, J.-H.; Shi, Z.J. Aqua-Net: An underwater sensor network architecture: Design, implementation, and initial testing. In Proceedings of the OCEANS 2009, Biloxi, MS, USA, 26-29 October 2009; pp. 1-8.

2. Masiero, R.; Azad, S.; Favaro, F.; Petrani, M.; Toso, G.; Guerra, F.; Casari, P.; Zorzi, M. DESERT Underwater: An NS-Miracle-based framework to design, simulate, emulate and realize test-beds for underwater network protocols. In Proceedings of the 2012 Oceans-Yeosu, Yeosu, Korea, 21-24 May 2012; pp. 1-10.

3. Le, S.; Peng, Z.; Cui, J.-H.; Zhou, H.; Liao, J. SeaLinx: A multi-instance protocol stack architecture for underwater networking. In Proceedings of the Eighth ACM International Conference on Underwater Networks and Systems, Kaohsiung, Taiwan, 11-13 November 2013.

4. Qiu, T.; Zhao, Z.; Zhang, T.; Chen, C.; Chen, C. Underwater Internet of Things in Smart Ocean: System Architecture and Open Issues. IEEE Trans. Ind. Inform. 2020, 16, 4297-4307. [CrossRef]

5. Rice, J.; Creber, B.; Fletcher, C.; Baxley, P.; Rogers, K.; McDonald, K.; Rees, D.; Wolf, M.; Merriam, S.; Mehio, R.; et al. Evolution of Seaweb underwater acoustic networking. In Proceedings of the OCEANS 2000 MTS/IEEE Conference and Exhibition. Conference Proceedings (Cat. No.00CH37158), Providence, RI, USA, 11-14 September 2000; Volume 2003, pp. 2007-2017.

6. Akyildiz, I.F.; Pompili, D.; Melodia, T. Underwater acoustic sensor networks: Research challenges. Ad Hoc Netw. 2005, 3, 257-279. [CrossRef]

7. Akyildiz, I.F.; Wang, P.; Lin, S.-C. SoftWater: Software-defined networking for next-generation underwater communication systems. Ad Hoc Netw. 2016, 46, 1-11. [CrossRef]

8. Cheremukhin, D.; Statsenko, L. Realizing underwater communication through magnetic induction. IEEE Commun. Mag. 2019, 53, 42-48. [CrossRef]

9. Demirors, E.; Sklivanitis, G.; Melodia, T.; Batalama, S.N.; Pados, D.A. Software-defined underwater acoustic networks: Toward a high-rate real-time reconfigurable modem. IEEE Commun. Mag. 2015, 53, 64-71. [CrossRef]

10. Campagnaro, F.; Francescon, R.; Casari, P.; Diamant, R.; Zorzi, M. Multimodal Underwater Networks: Recent Advances and a Look Ahead. In Proceedings of the International Conference on Underwater Networks \& Systems, Halifax, NS, Canada, 6-8 November 2017.

11. Diamant, R.; Casari, P.; Campagnaro, F.; Zorzi, M. Routing in multi-modal underwater networks: A throughput-optimal approach. In Proceedings of the 2017 IEEE Conference on Computer Communications Workshops (INFOCOM WKSHPS), Atlanta, GA, USA, 1-4 May 2017; pp. 205-210.

12. Saeed, N.; Celik, A.; Al-Naffouri, T.Y.; Alouini, M.-S. Underwater optical wireless communications, networking, and localization: A survey. Ad Hoc Netw. 2019, 94, 101935. [CrossRef]

13. Wang, J.; Kong, D.; Chen, W.; Zhang, S. Advances in Software-Defined Technologies for Underwater Acoustic Sensor Networks: A Survey. J. Sens. 2019, 2019, 1-13. [CrossRef]

14. Celik, A.; Saeed, N.; Shihada, B.; Al-Naffouri, T.Y.; Alouini, M.-S. A Software-Defined Opto-Acoustic Network Architecture for Internet of Underwater Things. IEEE Commun. Mag. 2020, 58, 88-94. [CrossRef]

15. Potter, J.; Alves, J.; Furfaro, T.; Vermeij, A.; Jourden, N.; Merani, D.; Zappa, G.; Berni, A. Software Defined Open Architecture Modem development at CMRE. In Proceedings of the 2014 Underwater Communications and Networking (UComms), Sestri Levante, Italy, 3-5 September 2014; pp. 1-4.

16. Torres, D.; Friedman, J.; Schmid, T.; Srivastava, M.B.; Noh, Y.; Gerla, M. Software-defined underwater acoustic networking platform and its applications. Ad Hoc Netw. 2015, 34, 252-264. [CrossRef]

17. Grassi, G.; Pesavento, D.; Pau, G.; Vuyyuru, R.; Wakikawa, R.; Zhang, L. VANET via Named Data Networking. In Proceedings of the 2014 IEEE Conference on Computer Communications Workshops (INFOCOM WKSHPS), Toronto, ON, Canada, 27 April-2 May 2014; pp. 410-415.

18. Zhang, L.; Afanasyev, A.; Burke, J.; Jacobson, V.; Claffy, K.; Crowley, P.; Papadopoulos, C.; Wang, L.; Zhang, B. Named data networking. ACM SIGCOMM Comput. Commun. Rev. 2014, 44, 66-73. [CrossRef] 
19. Kuai, M.; Haque, T.; Hong, X.; Yu, Q. A Named-Data Networking Approach to Underwater Monitoring Systems. In Proceedings of the 10th International Conference on Underwater Networks \& Systems, Arlington, VA, USA, 22-24 October 2015.

20. Bouk, S.H.; Ahmed, S.H.; Kim, D. NDN goes deep: Foreseeing the underwater named data networks. In Proceedings of the ACM Symposium on Applied Computing, Marrakech, Morocco, 4-6 April 2017; pp. 642-646.

21. Tariq, A.; Rehman, R.A.; Kim, B.-S. Forwarding Strategies in NDN-Based Wireless Networks: A Survey. IEEE Commun. Surv. Tutor. 2020, 22, 68-95. [CrossRef]

22. Liu, J.; Du, X.; Cui, J.; Pan, M.; Wei, D. Task-oriented Intelligent Networking Architecture for Space-AirGround-Aqua Integrated Network. IEEE Internet Things J. 2020. [CrossRef]

23. Basagni, S.; Di Valerio, V.; Gjanci, P.; Petrioli, C. Finding MARLIN: Exploiting multi-modal communications for reliable and low-latency underwater networking. In Proceedings of the IEEE INFOCOM 2017-IEEE Conference on Computer Communications, Atlanta, GA, USA, 1-4 May 2017; pp. 1-9.

24. Anand, A.; Dogar, F.; Han, D.; Li, B.; Lim, H.; Machado, M.; Wu, W.; Akella, A.; Andersen, D.G.; Byers, J.W.; et al. XIA: An architecture for an evolvable and trustworthy internet. In Proceedings of the 10th ACM Workshop on Hot Topics in Networks, Cambridge, MA, USA, 14-15 November 2011.

25. Ghodsi, A.; Shenker, S.; Koponen, T.; Singla, A.; Raghavan, B.; Wilcox, J. Intelligent design enables architectural evolution. In Proceedings of the 10th ACM Workshop on Hot Topics in Networks, Cambridge, MA, USA, 14-15 November 2011.

26. McCauley, J.; Harchol, Y.; Panda, A.; Raghavan, B.; Shenker, S. Enabling a permanent revolution in internet architecture. In Proceedings of the ACM Special Interest Group on Data Communication, Beijing, China, 19-23 August 2019; pp. 1-14.

27. Shen, X.; Gao, J.; Wu, W.; Lyu, K.; Li, M.; Zhuang, W.; Li, X.; Rao, J. AI-Assisted Network-Slicing Based Next-Generation Wireless Networks. IEEE Open J. Veh. Technol. 2020, 1, 45-66. [CrossRef]

28. Liang, C.; Yu, F.R.; Zhang, X. Information-centric network function virtualization over $5 g$ mobile wireless networks. IEEE Netw. 2015, 29, 68-74. [CrossRef]

29. Taneja, S.; Gupta, C.; Aggarwal, S.; Jindal, V. MFZ-KNN-A modified fuzzy based K nearest neighbor algorithm. In Proceedings of the 2015 International Conference on Cognitive Computing and Information Processing (CCIP), Noida, India, 3-4 March 2015; pp. 1-5.

30. Adeniyi, D.; Wei, Z.; Yongquan, Y. Automated web usage data mining and recommendation system using K-Nearest Neighbor (KNN) classification method. Appl. Comput. Inform. 2016, 12, 90-108. [CrossRef]

31. Kim, H.-W.; Cho, J.; Cho, H.-S. Topology-Aware Reinforcement Learning Routing Protocol in Underwater Wireless Sensor Networks. In Proceedings of the 2019 International Conference on Information and Communication Technology Convergence (ICTC), Jeju Island, Korea, 16-18 October 2019; pp. 124-126.

Publisher's Note: MDPI stays neutral with regard to jurisdictional claims in published maps and institutional affiliations.

(C) 2020 by the authors. Licensee MDPI, Basel, Switzerland. This article is an open access article distributed under the terms and conditions of the Creative Commons Attribution (CC BY) license (http://creativecommons.org/licenses/by/4.0/). 